with as recurrences in cases requiring repeated operation, and as first manifestations in some of the more obscure varieties, and for lupus erythematosus, of which I have had a conspicuous example in hand for the last two years. In that period scraping has been done seventeen times, at intervals of from twelve days to three months, in a woman aged 35 . The disease has lasted fourteen years, during the last ten of which I have frequently seen her. Some years ago her cheeks and nose were bright red, under a whole cuticle, and raised. She had been several times apparently nearly cured, after repeated cauterisation with a fine ileated point, but never got quite well. A very steady improvement has taken place under the seraping, which has sometimes had to be applied to minute miliary nodules no bigger than a millet-seed. For hæmostatic purposes I use a wire ring, set at an obtuse angle, mounted in a handle, and pressed firmly round the spot operated on; and, as soon as the scraping is done, solid nitrate of silver, rubbed into the raw surface. This effectually arrests or prevents bleeding, and secures for a few days an aseptic scab. In more extensive cases, requiring the sharn spoon, nitrate of silver is similarly applied, followed by a dressing of boracic lint or sublimated gauze, wet under waterproof tissue.

A year or two ago I used liquor calcis bisulphitis, a lotion that has been much praised for lupus and for the sores left after excision of rodent ulcer. But $I$ have not been able to find in it anything more than a convenient antiseptic, neither better nor worse than others in everyday use. I gave it a good trial also as a subcuticular injection into the lupus growth last referred to, before resorting to scraping, but it proved quite useless. What I claim for scraping is its thorough removal of morbid granulations, without waste by destruction of adjacent healthy structures, and the accuracy with which it can be done when the part is rendered bloodless by ring pressure.

\section{SCARLET FEVER AND ITS TREATMENT.}

Bx CLEMENT DUKES, M.D.LoND., M.R.C.P.LOND,

Physician to Rugby School, and Senior Physician to the Hospital of St. Cross, Rusby.

The Jocrnal of June 11th, 1887, contained a valuable article by Drs. Jamieson and Edington on "A Method of Prophylax:s, aud an Investigation into the Nature of the Contagium of Scarlet Fever.' They have proved that the specifie cause of scarlet fever is a bacillus, which they hare cultivated, and with which they have inoculated animals and produced scarlet fever. They have also shown that this bacillus occurs in the blood during the first three days of the fever that, later on, it is absent from the blood; and that it is found most extensively in the desquamating skin after the third week. They have, further, indicated a method by which this bacillus car be destroyed in the skin, and thus the spread of the infection of scarlet fever can be minimised, and the unprotected, even while residing in the same house, be safe from falling into its trammels.

But a still more important matter is the treatment and arrest of scarlet fever in each individual; for the first cry of a parent whose child has scarlet fever is, "What can you do to save my child; and how can you spare him from becoming mained for life by its sequelæ?" his second question being, "How can you prevent its spreading to my other children?" This second question Drs. Jamieson and Eding ton have answered. It is with the hope that I miy iuduce them to investigate the first question that I am writing this paper; for it has already bcen brought withici a measurable distance of beirg answered by Dr. Illingworth, of Accrington, who, in a letter in the Joursal for May 1st, 1886, stated that the biniodide of mercury $\left(\mathrm{Hg} \mathrm{I} \mathrm{I}_{2}\right)$ is a specific for scarlet fever. Recognising the importance of his letter in the use of mercury as a germicide, I resolved to administer the dring at the earliest opportunity. I have now given the $\mathrm{Hg} \mathrm{I}_{2}$ in several cases of scarlet ferer-with this result, that it not only arrests the fever, but it prevents the desquamation of the skin, or arrests it to such an extent that only a slight scurfiness of the skin of the hands and feet arises. If such be found to be invariably the case, will the bacilli of scarlet fever be found in the skin at all ; and, if not, will not the infectious period of scarlet ferer be thereby reauced to a few days only, and will not the sequelre of scarlet fever be absolutely prevented?

The $\mathrm{Hg} \mathrm{I}_{2}$ can be administered in the form of a pill or as a mixture of the liq. hyd. perchloridi c. pot. iodid. The only drawback to its use which I have at present found is that if it be given before the diagnosis is absolutely certain, the physician wili be apt to think, when he finds no desquamation taking place at the usual time, that the case was not one of scarlet fever. The drug prevents the desquamation of the epithelium of the tongue, as well as of the skin, and the throat rapidly heals under its use.
I was busy collecting facts when Drs. Jamieson and Ed̉ington's valuable paper appeared, and I should have waited until I had collected a sufficient number of instances before writing this paper had it not been for the desire that others, especially the above-named authors, would assist in establishing, or refuting, this treatment, for the experience of one individual is limited.

The benefit to be obtained from the use of $\mathrm{Hg} \mathrm{I}_{2}$ is far-reaching if it be reliable in all cases, for it not only prevents the desquamation of the skin, and thereby probably prevents the major part of the infectious nature of scarlet fever, but it will probably also be found that it obviates the necessity of keeping patients in bed for three weeks, which is the only safe rule hitherto, and isolated for five or six weeks, and will prevent the occurrence of the much-dreaded sequelæ.

The gist of the whole matter seems to be this: 1 , that if the bacilli of scarlet fever are only discoverva in the blood for about three days; 2 , that if the bacilli, after this date, chiefly occupy the desquamating cuticle; 3 , that if this desquamation can be prevented altogether by a medicine which destroys bacilli; 4 , then, in all probability, the infection of scarlet fever will only last a few days, and we are within a measurable distance of limiting the spread of scarlet fever, and of removing its fangs by preventing the sequelæ.

\section{NOTE}

\section{ON THE SO-CALLED "BACILLUS SCARLATINE" OF DRS. JAMIESON AND EDINGTJN.}

By WILLIANI R. SMITH, M.D., D.Sc., F.R.S.ED.,

Examiner in Forensic Jedicine and Hygiene, University of Aler leen, etc.

Ir having becn my lot for several years past to be more or less interested in the subject of zymotic diseases, having had until recently the medical charge of the Delancey Fever Hospital, and latterly taking some interest in the inquiry which has been promoted by the Local Government Board into the study of the etiology of scarlet fever, I naturally read with attention the paper published in the JouRNAL of June 11th last by Drs. Jamieson and Edington. It at once occurred to meputting asiaje other points in the paper to which exception might be taken - that the mode of growth of the so-called bacillus scarlatinæ was fundamentally opposed to the experience of our best observers in bacteriology on the life-history of pathogenic organisms in the following points :

a. That this organism was capable of forming a scum in a single night

$b$. That it grew abundantly in the presence of sereral species of septic organisms.

A further point which raised a doubt in my mind as to this organism being the cause of scarlet fever was the locality from which it was obtained. An objection, moreover, which I felt might fairly be urged to the conclusions arrived at was the absence of all control experiments with the scales of epidermis obtained either from the healthy or from certain irritative conditions of the skin.

With the view of supplementing the paper in this respect, I commenced a series of experiments with the scales of epidermis taken from the tibial surface of the leg, and, as might be expected, obtained growths of various kinds of organisms, amongst them being motile bacilli.

An opportunity presenting itself of obtaining good epidermic scales from a case of Sudamina, I made gelatine-plate cultivations with these in the ordinary way and with the usual precautions; in the course of twenty-four hours, evidence of growth was cle: rly manifest; after a further interval of twenty-four hours, in each of the plates a number of small whitish dots were seen, together with numerous points of clear, liquefied gelatine; upon the microscopical examination of these I found the former to consist of micrococci, and the latter of motile bacilli, which presented a striking resemblance to the representation given of the bacillus scarlatinæ in Fig. 10 of the plate illustrating the before-mentioned paper.

To procure a pure growth of this organism, I made further platecultivations, and succeeded in obtaining such a growth. From this, again, gelatine in test-tubes and broth in test-tubes were inoculated, and the characters of the bacilli thus obtained, both in microscopical specimens and in their different cultures, can best be given in the words descriptive of the "Bacillus scarlatinæ" (see JournaL, June 11 th, 1887, page 1265):

"Pods measuring $0.4 \mu$ in thickness and $1.2 \mu-1.4 \mu$ in length, most usually forming excessively long.jointed and curved leptothrix filaments, motile. Suwn on gelatine-plates it forms little points of 
liquefaction after several days. Sown in fluid broth and incubated, it rapidly forms a coherent parchment.like film, which is not easily broken up, and, even if the flask be well shaken, will yet float on the surface. Later, it develops an extraordinary deep wrinkling, which, if it be microscopically examined, is seen to be made up of a dense, felted network of bacillary filaments. Sown in test-tubes of Koch's jelly it rapidly liquefies it, but with no distinct growth-formation. The fluid thus formed is crowded with the motils bacilli, but a pellicle is not found until the liquefaction is well advanced."

From these observations I conclude that the organism provisionally named by Dr. Edington the "bacillus scarlatinæ," is in all probability nothing more than a common septic bacillus, which in former years was considered as belonging to the group of species of Bacillus subtilis of Cohn.

\section{THE PHARMACOLOGY OF DRUMINE. \\ BY JOHN REID, M.A., M.D., C.M.APERD. Melbourne, Australia.}

JUDGING from the correspondence which has demanded my attention on drumine, I may write without further prelude on its chemistry. A solution (aqueous) of drumini chloridum is used. With crystals of potassium ferrocyanide a purple coloration is obtained, which is better developed on applying heat, and appears in minute purple dots. If the experiment is conducted in a watchglass and the solution is allowed to evaporate, the purple deposit appears as a ring at the level of the original solution ; if evaporated by heat it is deposited all over. I think I may conclude from this that the purple substance is insoluble in water. I have, however, been unable to detect crystals under the microscope. This test is very delicate, and as negative results are obtained under like conditions with morphine bimeconate, and muriate, apomorphine chloride, atropia sulphate, pilocarpine nitrate, or a mixture of the whole, its value is evident. On adding drumine to the mixture and applying the test, the result at once appears.

Chromic acid absorbe; drumine chloride, and deposits needle-shaped and rhomboidal crystals. If heat is applied to the slightly deliquescad drumine-chromic acid, part becomes black, and microscopic crystals, consisting of feather-shaped units radiating from a centre (stellate) are almost the only occupants of the field. The darkened chromic acid of course resumes its pristine colour. A drop of water and chromic acid allowed to evaporate with drumine chloride deposits rhomboidal crystals. A bluish purple coloration may be seen at parts of the evaporated residue under the microscope, also when heat is applied to drumine-chromic acid. There is also evidence of a green chromic salt (chloride) independently of this.

Bichromate of potash crystallises with drumine chloride in rhom. boidal crystals. There are also sagittate crystals as if formed by cutting a triangular notch from one end of a rectangle, and fixing it to the other. These often occur in a stellate form. The like crystals are found in a mixed bichromate and picric acid solution. Crystals of potassium bichromate added to drumine solution produce a deep purple colour, developing better by the aid of heat; dark glistening green crystals also take the place of bichromate, and float on the solution. They may be evaporated; picric acid solution with drumine evaporates needles, also tree-shaped crystals-the branches and leaves being small and acicular-smaller than the ordinary drumine crystals. Picric acid salt is not freely soluble in water and salicylic acid. To prevent mistake, I again mention that cyanide of potassium in a neutral solution precipitates drumine from its chloride, and that drumine is insoluble in water; its chloride is freely soluble.

The dry reactions of drumine chloride are quite as interesting as the above. It is charred by heat, and so is the alkaloid; heated with picric acid a choking volatile substance arises along with white smoke, without causing the picric acid to melt. The sublimate was not yellow and not bitter; there was no carbonareous residue or detona. tion, as happens with picric acid alone (Watt's Dict. Chem. iv, 402, and Wurtz, Dict. de Chimie, Trinitrophenol). Bichromate of potash and heat merely char the drumine.

Contrasted with thallin, the re antions of drumuine are given above, while thallin causes a green colour with bichromate of potash, etc., in small quantities, and a white precipitate with excesa of the reagent, a yellow precipitate with picric acid not marked with drumine.

Having now reached this stage, I will refer to notices by "E. E.," Les Nouveaux Remèdes, 8 Mars, 1887, and Professor A. Ogston. "E. E." makes me precipitate the alkaloid from the chloride and again dissolve it (rather an expensive proceeding), and mentions my idea of two alkaloids. This is replaced by a belief in two types of crystallisation. Iodurated iodide of potassium ought to have, "above a certain concentration," added in order to indicate its power as a precipitant. Reference to my article in the Arstralian Med. Gazette, will show what were Dr. Schomburgk's statements, and what belonged to others. Professor Ogston's requires different treatment. I hope the learned and eminent professor will excuse me for finding fault with his method in endeavouring to arrive at a scientific conclusion. I gave simple directions to be followed, and also two tests for my alkaloid: 1, a microscopic ; 2, a physiological (quinine on one side of the tongue causes no taste if drumine chloride was previously applied). These would give a basis for work. It is better, I think, not to improve on an original experimenter's directions at the outset. With a drug acting on sensory nerves, as I have already pointed out, and as Charcot's writings will abundantly indicate, there are great difficulties to contend with. The heterogeneous nature of subjects allowed me to eliminate this varying factor. Witness a subject on whom morphine injection gave no permanent result, and a subject at random from the stage. But to return. My experiences with the decomposed drug coincide with those of Professor Ogston (I regret I am in a position to say so), but I am inclined to believe that the nervous factor induced the morphine symptom in Mr. Middleton; at least I have not observed it. My friend, Dr. P. Blaikie Smith, of Aberdeen, acknowledged the receipt of a spoiled specimen; Messrs. Savory and Moore also kindly informed me that a fungus had decomposed a specimen sent to them. I have given Messrs. Burroughs, Wellcome and Co., of Snow Hill, fully detailed directions for the preparation of drumine chloride, and I believe the pure alkaloid will soon be in the hands of the profession. The chemical, physiological and microscopic tests will enable them to judge of the specimens, as to whether they are identical with those which I have employed.

\section{CLINICAL MEMORANDA.}

THE HORIZONTAL POSITION OF THE MAMMILLA. IT is a common occurrence to read in published reports of cases that the "apex-beat was felt at 'such' a distance below the nipple," that statement conveying uo accurate idea as to the size of the heart.

The usual position of the mammilla is described in the text-books of anatomy as being about the fourth rib. The exact position was taken by me in 600 consecutive cases, 100 being children of 12 years and under, and 500 adults, of whom 326 were males and 174 females, the recumbent position being universally adopted.

Among males, 22 per cent. were over the 3rd space.

$\begin{array}{llll}37.7 \quad ", \quad " \quad & \text { 4th rib. } \\ 48.7 \quad ", & \text { " } \\ 11.4 & \text { " } & \text { 4th space } \\ 0.5 \text { per cent. } & \text { were over the } & \text { 3rd rib. }\end{array}$

Among females, 0.5 !per cent. were over the 3rd rib. 0.6 " " " 3rd space.

$17.8 \quad, \quad " \quad, \quad 4$ th rib.

$61.6 \quad " \quad$ " $"$ 4th space.

19.5 " ", ", 5th rib.

In children, the vast majority were over the 4 th rib. 1 per cent. being over the 3 rd rib.

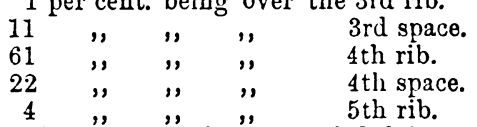

In all the 600 cases there was no instance of deficiency of nipple, and in only one (a male) of supernumerary nipples, two minor ones being partially developed two inches below the true ones. The fourt space is, therefore, the average position of the nipple in male and temale adults; the fourth rib in children.

II. T. Griffiths, M.D., M.R.C.P.

\section{QUIESCENT HEART DISEASE.}

I sexp you the following notes, as a good deal of interest has lately been evinced $r c$ long-standing cases of organic heart-disease with no symptoms, and their bearing on life-assurance.

Mrs. S., aged 71, suffered from acute rheumatism twenty-two years ago. From that date up to the present the only sickness of any importance has been an attack of erysipelas. She has led all along an extremely active existence as a midwife and nurse, a good amount of night-work and walking falling to her share.

The first intimation of anything amiss was about six months ago, when, after a short walk, she complained of pain in the cardiac region, with shortness of breath. Physical examination revealed oldstanding disease at the mitral orifice in the shape of regurgitation. As she has had no illness beyond the erysipelatous attack mentioned, 